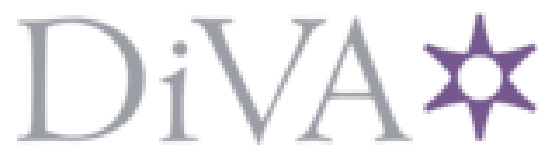

http://www.diva-portal.org

This is the published version of a paper published in .

Citation for the original published paper (version of record):

Raza, M R., Fiorani, M., Monti, P., Wosinska, L. [Year unknown!]

Demonstration of Dynamic Resource Sharing Benefits in an Optical CRAN.

Access to the published version may require subscription.

N.B. When citing this work, cite the original published paper.

Permanent link to this version:

http://urn.kb.se/resolve?urn=urn:nbn:se:kth:diva-192994 


\title{
Demonstration of Dynamic Resource Sharing Benefits in an Optical C-RAN
}

\author{
Muhammad Rehan Raza, Matteo Fiorani, Ahmad Rostami, Peter Öhlen, \\ Lena Wosinska, and Paolo Monti
}

\begin{abstract}
The next generation of mobile communication (i.e., 5G) will bring new challenges for the transport infrastructure, e.g., in terms of flexibility and capacity. The joint orchestration of radio and transport resources can help to address some of these challenges. One example is the possibility of reconfiguring the use of the transport network resources according to the spatial and temporal variations of the wireless traffic patterns. Using the concept of dynamic resource sharing, a limited pool of transport resources can be shared among a large number of radio base stations (RBSs), thus reducing considerably the overall deployment cost of the transport infrastructure. This paper proposes a provisioning strategy for a centralized radio access network with an optical transport whose wavelength resources can be dynamically shared among multiple RBSs. The proposed strategy utilizes a hierarchical software-defined networking control plane where a global orchestrator optimizes the usage of radio and transport resources. The benefits of the proposed strategy are assessed both by simulation and by experiment via an optical data plane emulator developed for this purpose. It is shown that the dynamic resource sharing can save up to $31.4 \%$ of transport resources compared to a conventional dimensioning approach, i.e., based on the overprovisioning of wavelength resources.
\end{abstract}

Index Terms-5G transport; Centralized radio access network; Dynamic resource sharing; Fronthaul; Optical network emulator; Software-defined networking.

\section{INTRODUCTION}

$\mathbf{T}$ he fifth generation of mobile networks (5G) will see early deployments as soon as 2017 and will be introduced on a wider scale around 2020 with the objective of providing a high data rate and nearly ubiquitous connectivity to humans and machines [1]. This new paradigm will be possible thanks to the introduction of new radio access technologies as well as to the adoption of new radio network architectures [2]. An example of new radio access technology is 5G-NX, which leverages new wireless solutions (i.e., massive beamforming and ultra-lean design) to provide very high capacity and energy efficiency [3].

Manuscript received March 22, 2016; revised July 2, 2016; accepted July 5, 2016; published July 29, 2016 (Doc. ID 261668).

M. R. Raza, M. Fiorani, L. Wosinska and P. Monti are with KTH Royal Institute of Technology, Department of Communication Systems, Electrum 229, SE-164 40 Kista, Sweden (e-mail: mrraza@kth.se).

A. Rostami and P. Öhlen are with Ericsson AB, Färögatan 6, SE-164 80 Kista, Sweden

http://dx.doi.org/10.1364/JOCN.8.000621
An example of a new radio network architecture is the heterogeneous network (HetNet), where small cells (SCs) are used together with macro cells (MCs) to provide higher wireless capacity. Another example of network architecture is the centralized radio access network (C-RAN), which centralizes the baseband processing functions of several radio cells to achieve better radio coordination and lower cost [4]. These changes in the radio access segment will require significant modifications in the transport network, i.e., the segment in charge of connecting the radio base stations (RBSs) with the evolved packet core (EPC) $[1,5]$. For example, the continuous radio densification with SCs will translate into a high number of RBSs to be connected to the transport infrastructure. Another example is the Common Public Radio Interface (CPRI) protocol, used in C-RAN to connect the RBSs to the centralized baseband resources, which puts high-capacity requirements on the transport networks [1]. Overall, the future 5G transport networks will need to be highly scalable to efficiently support both an increasing number of RBSs and a higher capacity per RBS. Consequently, the upcoming $5 \mathrm{G}$ scenarios will require very flexible ultrahigh capacity transport networks, making the cost effectiveness of the network infrastructure of primary importance.

Radio and transport networks are currently controlled and provisioned separately. The transport infrastructure in most of the cases is designed to support peak wireless traffic requirements, i.e., a technique known as overprovisioning. This way of designing and deploying the transport network is simple but not efficient due to the fact that the user traffic demand, in terms of wireless capacity, usually varies significantly in both space and time [6]. As a result, in large network scenarios, only a subset of all the RBSs needs to be active at the same time, and an overprovisioned transport network is most of the time underutilized.

A promising solution for reducing the cost of the transport network is to employ an integrated control architecture where the radio and transport resources are jointly orchestrated. This enables one (i) to dynamically turn on/off RBSs (i.e., according to the wireless traffic variations) and (ii) to reconfigure accordingly the transport network so that only a limited pool of transport resources can be dynamically shared to serve a large number of RBSs. Thanks to this orchestration, it is possible to design provisioning strategies that enable a much more efficient use of the transport network infrastructure. 
Software-defined networking (SDN) is a control architecture that has the potential to enable the joint orchestration of radio and transport domains [7]. In a hierarchical SDN control plane, the radio and the transport network can be managed by two separate controllers, while a global orchestrator jointly allocates the radio and transport resources. A first demonstration of this concept has been recently presented in Ref. [7], where a hierarchical SDN control plane was deployed to improve the efficiency of using transport resources. The authors assume a C-RAN based on an optical dense wavelength division multiplexing (DWDM) transport network. The proposed orchestration framework is able to dynamically turn on/off RBSs and to reconfigure the DWDM network so that a reduced number of wavelengths is needed to serve several RBSs. However, the work in Ref. [7] considers an experimental setup with only four RBSs to show the feasibility of the concept.

This paper extends the previous work with two contributions. First, we propose a provisioning strategy based on dynamically shared transport resources that can be utilized in a C-RAN scenario with an optical DWDM transport. The proposed strategy relies on the use of an orchestrator able to (i) switch on/off RBSs according to the wireless traffic requirements and (ii) redirect the wavelength resources to serve only those RBSs currently active. Second, we developed an optical network emulator (ONE) in order to experimentally evaluate the hierarchical SDN control plane solution proposed in Ref. [7] and assess its scalability in a realistic network scenario. We then prove both via simulation and emulation that with the proposed provisioning strategy, it is possible to save up to $31.4 \%$ of transport resources compared to a conventional overprovisioning-based approach.

\section{RELATED WORK}

The joint orchestration of different technology domains has been recently proposed in the literature as one possible way to optimize the utilization of the network resources.

The majority of the works published so far by both academia and industry are focused on the joint orchestration of transport and information technology (IT) resources. For example, the work in Ref. [8] presents the concept of joint orchestration of transport and cloud resources to provide intra/inter-data center (DC) connectivity and to establish end-to-end paths through heterogeneous networks. This work is extended in Ref. [9] with several different controllers coordinating with each other through an orchestrator in order to provide a seamless migration of virtual machines in a multi-layer and multi-domain network. In these works, the benefits of the joint orchestration of transport and cloud domains have been quantified in terms of an improved efficiency in the use of the transport and cloud resources. Another interesting work is the one presented in Ref. [10], where the authors propose an architecture for the joint orchestration of cloud and transport domains in order to dynamically deploy and connect network functions of newly instantiated virtual networks. Also in this case, the advantages of the joint orchestration are measured in terms of an improved efficiency in the use of the transport and cloud resources and thus a potential reduction of the cost of the infrastructure. Moreover, in the work presented in Ref. [11], a joint orchestration technique unifying the control of cloud and transport resources has been designed and implemented as part of the UNIFY project [12]. This technique can be used for reducing the cost of the transport and the cloud infrastructure.

Although the joint orchestration of transport and cloud domains has gained a lot of interest in the research community, so far, not much work has been done to explore the potential benefits of the joint orchestration of transport and radio resources. The authors of Refs. [13] and [14] propose a technique for the virtualization of the EPC in a mobile network and an SDN-based control architecture that can be used to dynamically configure the transport network so that the traffic is dynamically routed toward the closest available virtualized EPC. The authors state that this solution can improve utilization of the transport resources and reduce the cost of the transport infrastructure by $7.7 \%$. Finally, the works in Refs. $[7,15,16]$ present a proof of concept depicting the benefits (in terms of transport resources usage) of a joint orchestration of radio and transport resources in a C-RAN scenario using a reconfigurable DWDM transport. However, the advantages of the proposed solution in a realistic network scenario have not been evaluated yet.

\section{System Architecture}

The mobile access network architecture considered in this paper is based on the C-RAN concept (Fig. 1). The network architecture includes two parts, i.e., the data and the control plane.

\section{A. Data Plane}

The data plane is based on the interconnection of DWDM rings. The RBSs in the network, i.e., macro base stations (MBSs) and SCs, are served by the access rings via access

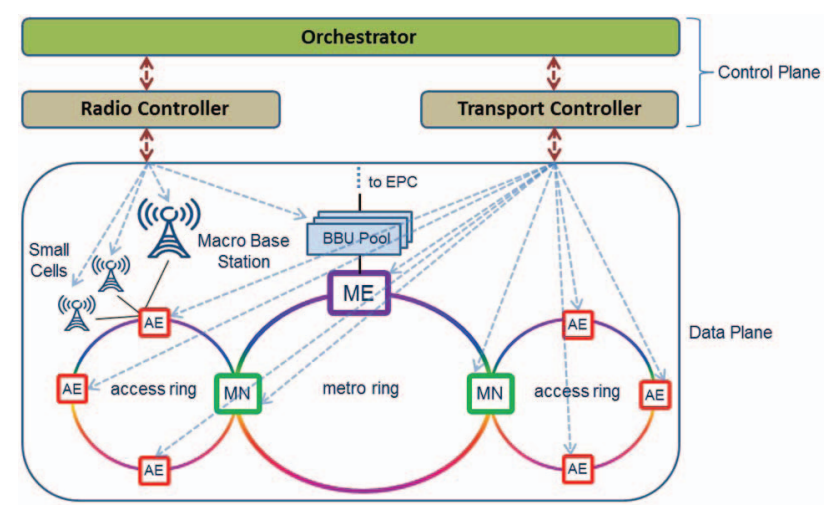

Fig. 1. Mobile access network architecture showing both the data and the control plane. AE: access edge; $\mathrm{MN}$ : metro node; $\mathrm{ME}$ : metro edge; BBU: baseband unit; EPC: evolved packet core. 
edge (AE) nodes, to which the RBSs are connected. ${ }^{1}$ The access rings are geographically distributed to cover a given region comprising different geo-types (e.g., residential, business, city center). These geo-types have different wireless capacity requirements that vary in time. As a result, each access ring is characterized by its own daily traffic profile. The access rings are connected to the metro edge (ME) node via a metro ring and the metro nodes (MN). The ME hosts a BBU Pool where the baseband processing takes place. The BBU Pool is in turn connected to the EPC. Each time an RBS is activated, two connections need to be established in the network: one from the RBS to the BBU Pool, and one from the BBU Pool to the EPC. The work presented in this paper targets the connection establishment problem in the fronthaul segment only (i.e., the part of the transport network connecting the RBSs and the BBU Pool) and does not consider the backhaul segment (i.e., the part of the transport network connecting the BBU Pool and the EPC). Each time an RBS is deactivated, both connections are released.

With the architecture presented in Fig. 1, the upstream traffic coming from the MBSs and SCs is aggregated at the $\mathrm{AE}$ nodes and forwarded to their respective $\mathrm{MNs}$ via the access rings. The MNs aggregate the traffic from the access rings and deliver it to the $\mathrm{ME}$ via the metro ring. In the downstream direction, when reaching a specific RBS, the nodes are traversed in the reverse order, i.e., from the $\mathrm{ME}$ to the $\mathrm{MN}$ and finally to the $\mathrm{AE}$ to which the RBS is connected.

The node architecture of the $\mathrm{ME}, \mathrm{MN}$, and $\mathrm{AE}$ is presented in Fig. 2. We consider this architecture based on wavelength-selective switches (WSSs) in order to be able to dynamically assign wavelength resources in the network (i.e., between the $\mathrm{AEs}$ and the $\mathrm{ME}$, and vice versa) according to the traffic demand. The ME architecture consists of two WSSs whose common ports are connected to the metro ring, while the tributary ports are connected to the ports of the BBU Pool. This architectural choice allows the $\mathrm{ME}$ to connect to an RBS by traversing the metro ring in the clockwise or the counter-clockwise direction. Each BBU port in the BBU Pool is equipped with a wavelengthtunable transponder (TP). The MN has two WSSs interconnected via one of the tributary ports. The other tributary ports carry the traffic to/from the access rings, while the common port of each WSS carries the traffic to/from the metro ring. The $\mathrm{AE}$ consists of four WSSs. Three of them are interconnected in a ring, while the fourth one is connected directly to the RBSs. This architectural choice allows each RBS to be reached by traversing the access ring in the clockwise or the counter-clockwise direction. Moreover, each RBS is equipped with a wavelengthtunable TP for transmitting/receiving the optical signal.

In this paper, we assume that the traffic in the uplink and the downlink directions is carried over two separate

\footnotetext{
${ }^{1}$ The paper assumes a centralized radio access network (C-RAN) architecture. For this reason, the term radio base station (RBS) used throughout the paper refers to a system consisting only of a number of remote radio units and antennas placed at the cell site.
}
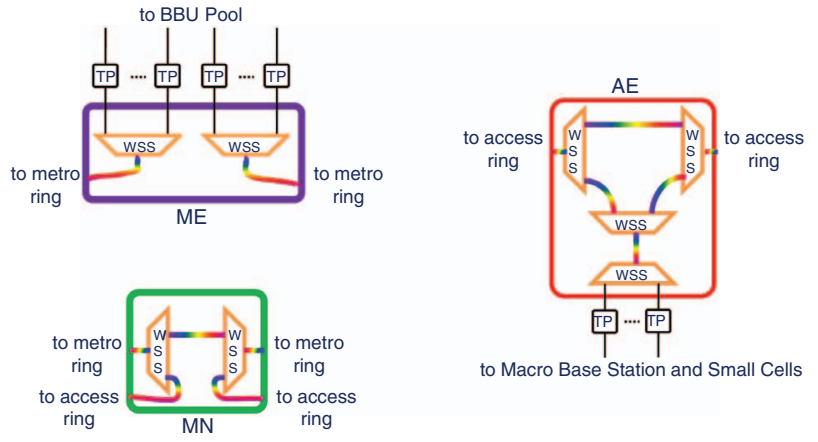

to Macro Base Station and Small Cells

Fig. 2. Node architecture of the ME, the MN, and the AE.

data planes, each one with its own fiber system with unidirectional transmission in each fiber, using the architecture described earlier. Finally, it is worth mentioning that with the node architectures presented in Fig. 2, it is possible to serve only as many RBSs as the maximum number of wavelengths available in all the fiber links in the network (assuming one has the same type of fiber installed in all links in the access and metro rings). When the number of RBSs to be connected to the BBU Pool exceeds this number, additional fibers need to be installed in the network, each one requiring an extra layer of WSSs in all the network nodes.

\section{B. Control Plane}

This paper considers the control plane architecture depicted in Fig. 1. An orchestrator sits on top of the radio and transport controllers. It is in charge of deciding which actions should be performed in the network (e.g., when/ where to switch on/off RBSs in the wireless segment, and when/where to establish/release optical connections in the transport segment). Once the orchestrator makes a decision, the information about which network resources to activate/deactivate is sent to the respective controllers for execution.

The radio controller is responsible for monitoring how resources are used in the wireless segment of the network, i.e., RBSs and the BBU ports in the BBU Pool. The radio controller sends this information to the orchestrator, which accordingly decides which RBSs should be turned on/off at any time of the day. The radio controller is also responsible for turning on/off RBSs when the orchestrator decides to do so.

The transport controller is in charge of configuring the optical resources (WSSs and/or TPs) in the ME, MNs, and AEs. More specifically, the main task of the transport controller is to establish/remove optical connections between the AEs and the ME according to the orchestrator's decision to activate/deactivate a given RBS. The transport controller sends back a positive acknowledgment to the orchestrator if a connection has been established/removed successfully. Otherwise, it notifies the orchestrator about the unsuccessful attempt. When a new optical connection needs to be established between a given RBS and a BBU 
port, the transport controller is also in charge of deciding which route and wavelength the connection should use, i.e., of solving the routing and wavelength assignment (RWA) problem.

With the control architecture just described, it is possible to dynamically adapt the use of the network resources to the traffic levels of each geo-type. For example, if, during the daytime, one or more business areas experience an increase of the traffic demand, the orchestrator may decide to activate more SCs (and consequently establish more optical connections) where needed. This trend may be different during the evening/night, i.e., less traffic demand in the business areas, but an increase of the traffic demand in the residential sites. The orchestrator may then decide to deactivate some of the SCs in the business areas and to activate more SCs in the residential sites. As a result, some of the optical connections provisioned in the business areas can be re-routed to serve the users in the residential sites. The orchestrator can take these decisions in real time according to the traffic information provided by the radio controller. This concept is known as dynamic resource sharing and allows for a more efficient use of the resources. The next section elaborates more on the benefits of dynamic resource sharing and provides more details on how it can be applied in the data plane of the C-RAN architecture under examination.

\section{Dynamic Resource Sharing and Provisioning STRATEGY}

In a C-RAN scenario, an approach based on overprovisioning can be applied to guarantee that enough transport resources are always available when an RBS is activated. With this strategy, the number of TPs deployed at the ME is the same as the total number of RBSs in the network. However, as was made clear in the previous section, when looking at the traffic requirements at different times in a day, only a subset of all the RBSs might need to be active at the same time. Following this reasoning, it might be preferable to deploy only a subset of the TPs required by an overprovisioning-based approach and share them dynamically as needed to connect RBSs and BBU ports, i.e., use a dynamic resource-sharing strategy to provision fronthaul connections. As already explained earlier, each new fronthaul connection requires the establishment of an optical connection (i.e., a lightpath) between an RBS and a BBU port. For this reason, a vital part of every dynamic resource-sharing strategy is the ability to dynamically set up and tear down lightpaths as needed. The lightpath provisioning strategy proposed in this paper is described next.

The idea is to try to connect the ME to the newly activated RBS using the shortest path with the available wavelength resources chosen between the clockwise and the counter-clockwise options. In order to do so, when a given RBS needs to be activated, the orchestrator performs the following set of actions (Fig. 3). First, it asks the transport controller to compute a counter-clockwise path, i.e., from the left WSS at the ME to the AE (where the RBS

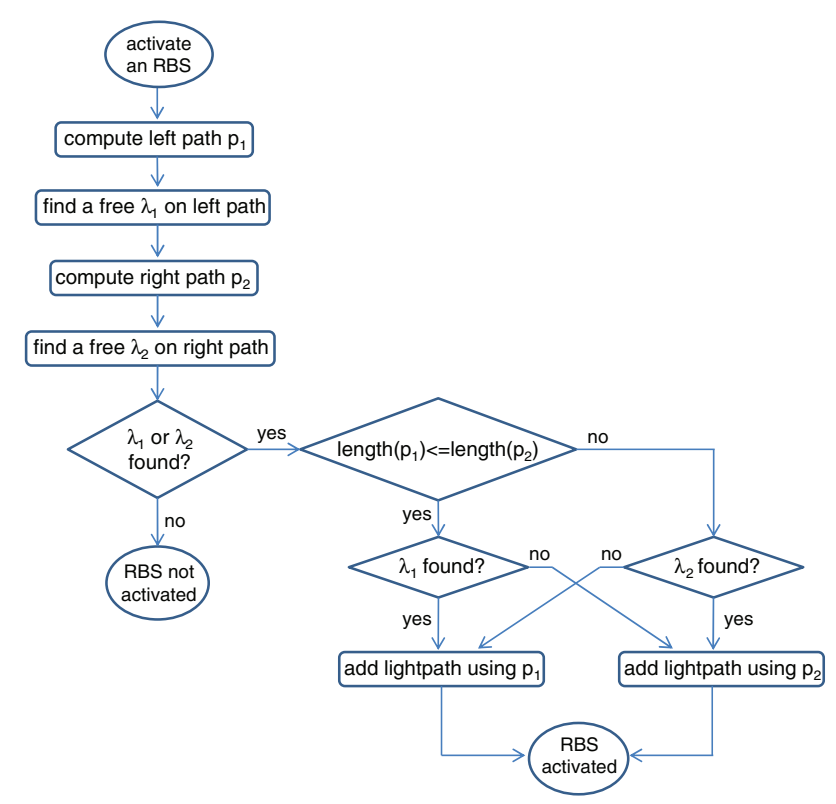

Fig. 3. Flowchart describing the actions performed by the orchestrator when an RBS needs to be activated.

is connected) by traversing the metro and access rings in the counter-clockwise direction. If such a path is available, the transport controller is also asked to identify a free wavelength along the computed path using a first-fit strategy. This wavelength cannot be already in use on any of the links of the computed path (i.e., the wavelength clash constraint is enforced). If this operation is also successful, the orchestrator stores both the wavelength ID and the computed path. The same set of operations is then performed for the clockwise path, i.e., from the right WSS at the ME to the AE (where the RBS is connected) by traversing the metro and access rings in the clockwise direction. If no paths and/or no free wavelengths are found for both the clockwise and counter-clockwise options, the RBS cannot be activated.

Otherwise, the orchestrator compares the length of both path options and instructs the transport controller to connect the $\mathrm{ME}$ and the $\mathrm{AE}$ with a lightpath traversing the shorter path between the two. At this point, it might be possible that the shorter path does not have a wavelength available (i.e., this is because of the "OR" condition used to the check the wavelength availability in the flowchart in Fig. 3). If a wavelength is not available on the shorter path, the other (i.e., longer) path option is selected. When the transport controller acknowledges that the lightpath has been established successfully, the orchestrator activates the corresponding RBS by sending the required commands to the radio controller. ${ }^{2}$

${ }^{2}$ In the system architecture under examination, it is assumed that the distance between the ME and any of the AEs does not exceed the distance constraint imposed by the CPRI protocol [17]. However, the proposed approach can be easily extended to take this limit into account if it needs to be applied to other C-RAN architectures, e.g., with a large-scale meshed data plane topology. 
Using the lightpath provisioning strategy just described, the dynamic resource-sharing approach evaluated in the paper works as follows. Since MBSs are always kept active to provide coverage over the entire area, the first operation performed by the orchestrator is to establish a fronthaul connection to all the MBSs in the network. On the other hand, SCs in the network can be turned on and off dynamically to accommodate the variation in the traffic requirements in all the areas covered by the access rings.

If, based on the information from the radio controller, the orchestrator realizes that the traffic requirement on an $\mathrm{AE}$ exceeds the capacity provided by the currently active RBSs, it activates one extra SC connected to the corresponding $\mathrm{AE}$. This is done by asking the transport controller to establish another lightpath between the ME and the corresponding $\mathrm{AE}$ and instructing the radio controller to turn on the corresponding SC if the lightpath has been established successfully. If the traffic requirements are still not met, additional SCs are activated at the $\mathrm{AE}$ following the same procedure.

At any point in time, based on the feedback from the radio controller, the orchestrator may also realize that the capacity provided by the number of currently active SCs exceeds the traffic requirement at a given $\mathrm{AE}$. In this case, the orchestrator deactivates one or more $\mathrm{SCs}$ via the radio controller and instructs the transport controller to tear down the corresponding fronthaul connections.

With this procedure, it is possible to activate and deactivate SCs connected to AEs based on the current traffic requirements.

\section{Optical Network Emulator}

This section presents the ONE developed to experimentally validate the simulation results of the dynamic resource-sharing strategy proposed in Section IV. One of the major motivations for us to design the ONE was to provide an experimental testbed that can be used to evaluate the performance of very large networks and test the scalability of the considered hierarchal SDN control plane.

The ONE is used to emulate the optical transport data plane, but it can be controlled, on the other hand, by a real control plane instance. For the experimental work presented in this paper, the ONE is connected to the control plane presented in Ref. [7]. This control plane instance is based on OpenDaylight (ODL) [18], and it has been extended with a number of new functions to enable the control of optical network elements [7]. One of the most important extensions is the design and implementation of a southbound plugin in ODL for controlling TPs and WSSs. This interface is named the lambda interface, and it is used to send commands to TPs and WSSs in order to configure them.

In the ONE, TPs and WSSs have been emulated by means of packet-based Open vSwitches (OVSs) [19] while the mininet software [20] has been used to create a data plane topology consisting of several OVSs. Since the lambda interface of ODL has been designed to send the commands to the real TPs and WSSs, the emulated TPs and WSSs must be able to understand these commands in order to properly configure lightpaths inside the network. However, the OVSs are only able to understand OpenFlow (OF) [21] commands. For this reason, we implemented a translation layer in Python that translates the commands from the lambda interface (e.g., laseron, laseroff, setchannel, etc.) into the corresponding OF commands. These commands are then sent to the OF controller, which inserts the corresponding flow entries in the underlying OVS. Since we need to have a translation layer and an OF controller for each emulated element in the network (i.e., TP and WSS), we decided to choose a very lightweight OF controller, i.e., $P O X$ [22], in order to ease the burden on the operating system. The emulation architectures of TP and WSS are shown in Figs. 4 and 5.

In order to emulate the transmitting functionality of a real TP that assigns a (tunable) wavelength to an incoming flow, a virtual local area network (VLAN) tag is inserted into each incoming packet that enters the emulated TP, e.g., shown by the red color in Fig. 4. Different VLAN tags are assigned to the packets corresponding to lightpaths with different wavelengths, e.g., VLAN tag 101 for wavelength 1 , VLAN tag 102 for wavelength 2, etc. When the emulated TP receives a command from the lambda interface to tune the TP to a specified wavelength, the OF controller installs a flow entry in the OVS to tag all ingress packets with the corresponding VLAN tag. When a TP needs to be turned off or tuned to another wavelength, the corresponding flow entry is removed or modified accordingly. For emulating the receiving functionality of a TP, a flow entry is inserted in the OVS for stripping the VLAN tag from all incoming packets and forwarding them to the output port. This flow entry is installed proactively when the network is initialized, since the TP acts as a broadband receiver during the whole lifetime of the emulation experiment. However, other flow entries are installed reactively when the TP is tuned to a specified wavelength after receiving the corresponding command from the lambda interface of the ODL.

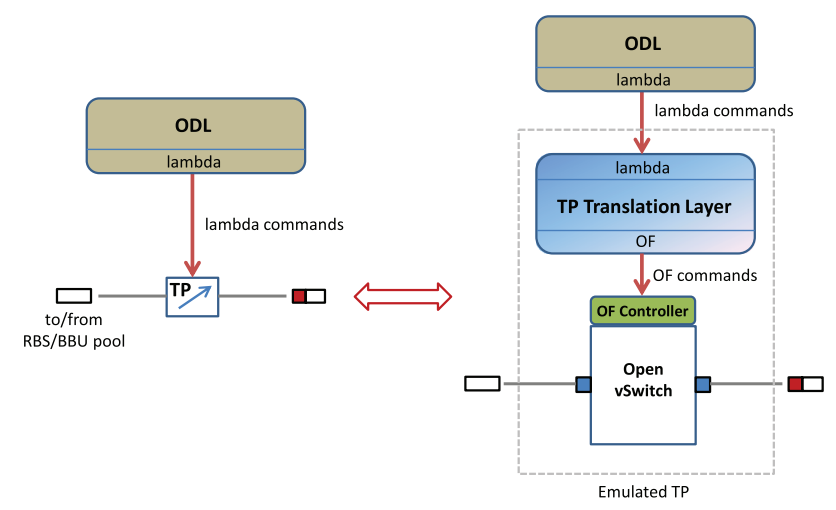

Fig. 4. Emulation architecture of a TP depicting how the commands from the lambda interface of the ODL controller are translated into OF commands for OVS. 
Similarly, the emulated WSSs translate the commands from the lambda interface (i.e., meant for a real WSS) into OF commands to insert (or remove) the corresponding flow entries in the OVS. The flow entries in this case refer to forwarding the incoming tagged packets to a specified WSS port, with the VLAN tag corresponding to the wavelength mentioned in the command from the lambda interface. Moreover, the translation layer in this case takes into consideration the fact that each wavelength can be used only once on the tributary ports of the WSS. As shown in Fig. 5, packets with different VLAN tags (shown by different colors) at the tributary ports are forwarded to the common port by OVS.

It is worth mentioning that the emulation work considered in this paper takes into account only TPs and WSSs because these are the only optical devices used in the transport network architecture under examination. However, more complex optical elements (e.g., filters, combiners, splitters, wavelength converters) can also be emulated, if needed, with an architecture similar to the one used to emulate TPs and WSSs, i.e., OVS and a translation layer. In the next section, we describe the scenario under which the ONE is used for the experimental validation of our simulation results.

\section{Description of the Scenario}

We analyze the $5 \mathrm{G}$ dense urban scenario described in Refs. [23,24], i.e., an area of $2 \mathrm{~km} \times 2 \mathrm{~km}$ where the wireless coverage is guaranteed using $35 \mathrm{MBSs}$, each one connected to its own AE. A layer of SCs, i.e., $N$ for each AE, is used to provide extra capacity where/when needed, as shown in Fig. 1. As a result, every AE is connected to $(N+1)$ RBSs, each one equipped with one TP. The area under consideration is covered by seven access rings. Each access ring has five $\mathrm{AEs}$, while a metro ring consisting of three MNs connects all access rings to the ME.

Two different traffic models have been considered for modeling the variation of the wireless capacity requirements in the access rings. The first model is derived from Ref. [25], and it describes the average daily traffic variation

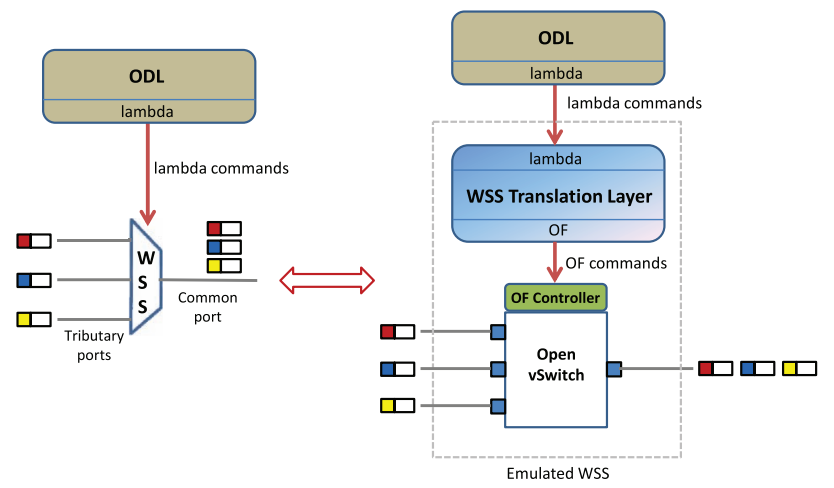

Fig. 5. Emulation architecture of a WSS depicting how the commands from the lambda interface of the ODL controller are translated into OF commands for OVS.

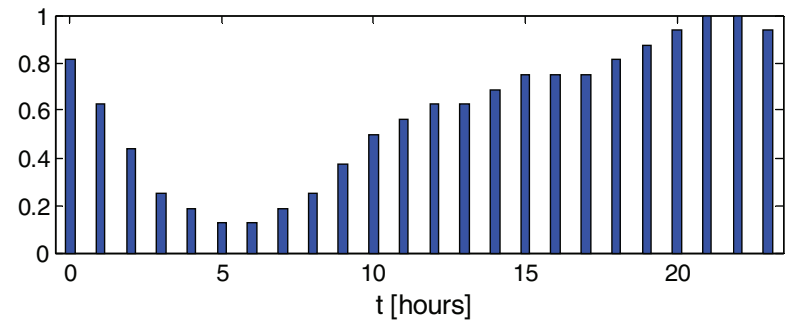

Fig. 6. Traffic profile (normalized to the peak value) depicting the average daily traffic variation in Europe [25].

in a European city. The traffic profile is depicted in Fig. 6 and has been obtained by averaging the data collected by several European mobile network operators participating in the EARTH project [25]. In each access ring, the daily temporal variations of the wireless capacity requirements (i.e., during 24 hours) are modeled using this traffic profile. On the other hand, the spatial variations of the wireless capacity (e.g., different geo-types have different requirements) are modeled by shifting the traffic profile in each access ring by three hours. A three-hour time shift is selected to randomize the capacity requirements at each hour in the seven access rings. Different time shifts have also been considered, providing in most cases very similar results to the ones that will be discussed in Section VII.

The second model is taken from Ref. [6], and it describes the daily traffic variation in a large Asian city for different geo-types, e.g., residential, office, and transport areas. These traffic profiles were obtained by elaborating the data collected by an Asian mobile network operator over a period of one month [6]. The traffic profiles for different geo-types are presented in Fig. 7. The spatial variations of the wireless capacity are modeled by randomly assigning a traffic profile of a different geo-type to each access ring, whereas the temporal variations are modeled by the specific traffic profile assigned to the access ring (i.e., according to Fig. 7).
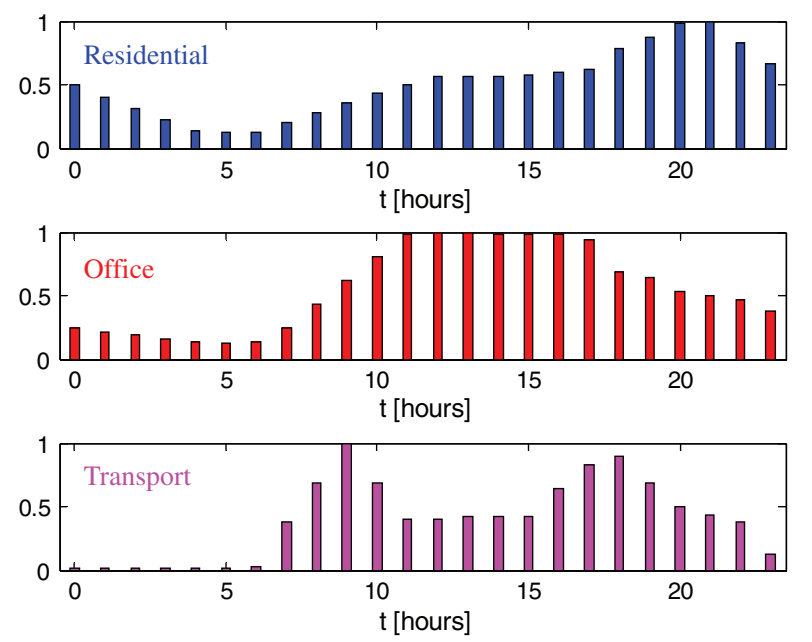

Fig. 7. Traffic profiles (normalized to the peak value) depicting the daily traffic variation in a large Asian city for residential, office, and transport areas [6]. 
Unless stated otherwise, the traffic profile of each access ring has a maximum capacity value of $1 \mathrm{Gbps} / \mathrm{km}^{2}$ [23]. We assume that the maximum capacity value provided by an RBS is equal to $100 \mathrm{Mbps} / \mathrm{km}^{2}$ for an MBS and $50 \mathrm{Mbps} / \mathrm{km}^{2}$ for an SC. For the transport segment, we assume that each fiber link in the access and metro rings comprises 96 wavelengths with $50 \mathrm{GHz}$ channel spacing, while TPs located at the AEs and at the ME operate at $10 \mathrm{Gbps}$ (to support the capacity required by the fronthaul traffic) with an on-off keying modulation format.

In each simulation experiment, the traffic profile of each access ring is sampled every hour in order to derive the traffic profile of each $\mathrm{AE}$, i.e., the capacity value as a result of each sampling is randomly divided among the five AEs of each access ring. The traffic profile of each $\mathrm{AE}$ is then used to count the number of RBSs that need to be activated at each hour in order to provide the required capacity. Please note that since the MBSs are always active to guarantee wireless coverage, only SCs are activated/deactivated to follow the temporal/spatial variation of the wireless capacity requirements. In the scenario just described, it might happen that some AEs might become overloaded before new SCs are turned on (i.e., during the time period between two consecutive measurements). This can be avoided by defining appropriate thresholds while monitoring the wireless traffic variations so that additional SCs can be activated proactively in order to avoid capacity starvation. On the other hand, these thresholds need to be carefully decided in order to find the right trade-off between the quality of service delivered to the users and the achievable gains coming from a dynamic resource-sharing strategy.

Following the explained rationale, at the beginning of each experiment, all the MBSs are activated, i.e., one lightpath is established from the ME to all the AEs. Then, based on the traffic profile of each $\mathrm{AE}$, a number of SCs are activated/deactivated to match the $\mathrm{AE}$ traffic profile. This number is computed by comparing the value of the $\mathrm{AE}$ capacity requirement (at any given hour) with the maximum capacity that can be provided by the MBS and the SCs that were eventually turned on in previous time intervals. If this difference in capacity is greater than zero, this means additional SCs need to be turned on. If the difference in capacity is lower than zero, one (or more) SC needs to be turned off. The exact number of SCs to be turned on/off is obtained by computing the ceiling of the ratio between the difference in capacity and the value of the maximum capacity provided by an SC. Whenever an SC is activated, a lightpath needs to be established between the $\mathrm{ME}$ and the corresponding $\mathrm{AE}$. Conversely, when an $\mathrm{SC}$ is deactivated, the corresponding lightpath is torn down. Lightpaths are established and removed according to the dynamic provisioning strategy described in Section IV.

\section{Results}

In this section, we first describe the experimental setup used in the emulation experiments. Then, we present the numerical results obtained both by simulation and emulation, which depict the benefits of the proposed dynamic resource provisioning strategy as compared to overprovisioning.

\section{A. Experimental Setup}

The simulation results have been experimentally validated using the ONE (described in Section V). The experimental setup for the emulations is shown in Fig. 8. Note that in our emulation experiments, we employ the data plane architecture with seven access rings and one metro ring described in the previous section. However in Fig. 8, we show a simplified version of the data plane architecture for the sake of clarity. In order to mimic the radio controller, we use a MATLAB script that generates a text file containing the daily traffic profiles in each of the AEs in the network. These daily traffic profiles are generated as described in the previous section. Moreover, in order to mimic the functionalities of the orchestrator, we employ a script in Java that reads the daily traffic profiles from the text file to identify which SCs should be turned on/off at each hour of the day. Accordingly, our script in Java employs the provisioning strategy presented in Section IV to identify the lightpaths that need to be established/torn down in the optical network in order to connect all the active RBSs to the BBU pool. The script in Java sends the commands to ODL in order to establish/teardown the selected lightpaths in the emulated optical data plane. The lightpaths are routed in the optical network using the path computation element (PCE) of the ODL controller.

In Fig. 9, we present some screenshots of an emulation experiment. These screenshots have been taken while running the emulation experiment for $N=2$.

Fig. 9(a) shows the running ODL controller while creating the data plane architecture described in the previous section. The entry highlighted with a red box shows (as an example) that a TP with name tp -3 has been added by ODL in the transport network. Note that the data plane architecture is provided to ODL by using an XML file which contains the information about network topology consisting of TPs and WSSs. The ODL runs a PCE algorithm over the provided topology and sends the commands to the underlying emulated TPs and WSSs through the lambda interface in order to add/remove the flows, as needed. The mininet also reads from the same XML file in order to generate exactly the same architecture, with an OVS corresponding to each TP and WSS in the XML file. It assigns an OF controller to each of the OVSs, and afterwards, the Python scripts for the TP or WSS translation layer are started for each OVS depending on whether the OVS corresponds to a TP or a WSS.

Figure 9(b) shows some of the nodes in which flow entries have been installed by the OF controller after receiving commands from the lambda interface in ODL. The entry highlighted in red shows the proactive flow entry that is inserted in all the TPs during initialization in order to make them work as a receiver. This entry results in OVS stripping the VLAN tag from all packets arriving at port 1 and forwarding them to port 2 . The entry highlighted in 


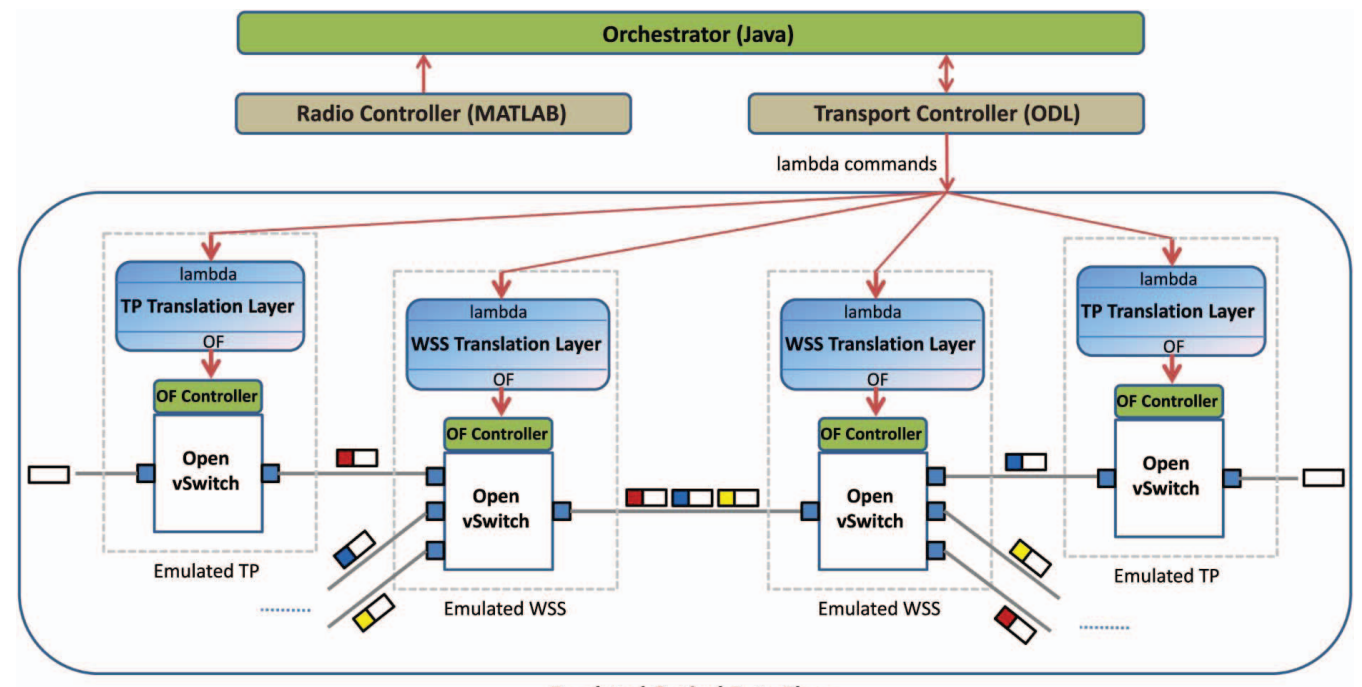

Emulated Optical Data Plane

Fig. 8. Experimental setup for emulation.

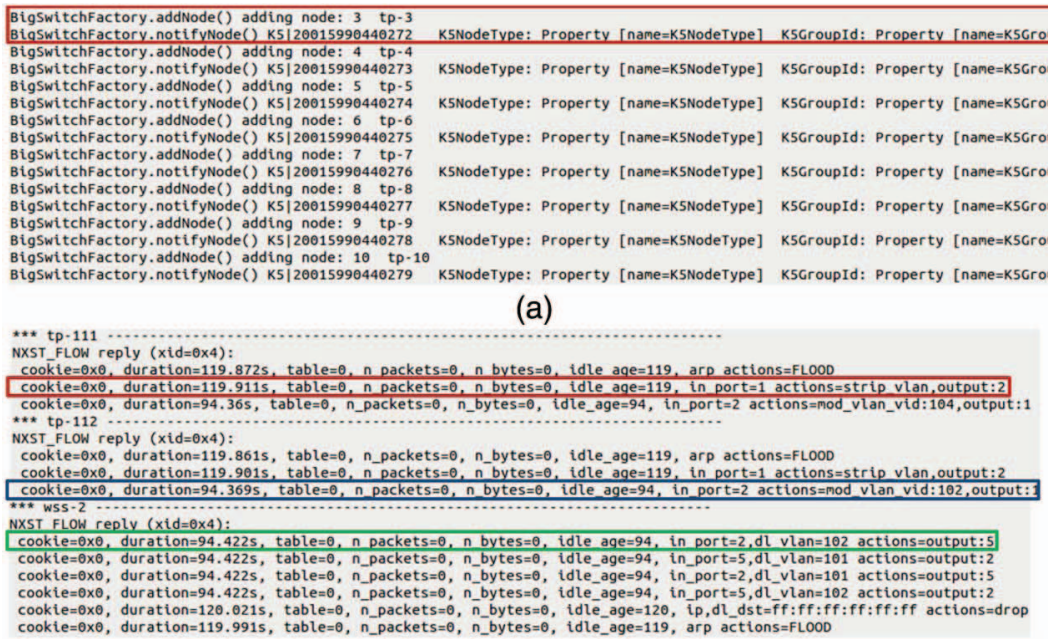

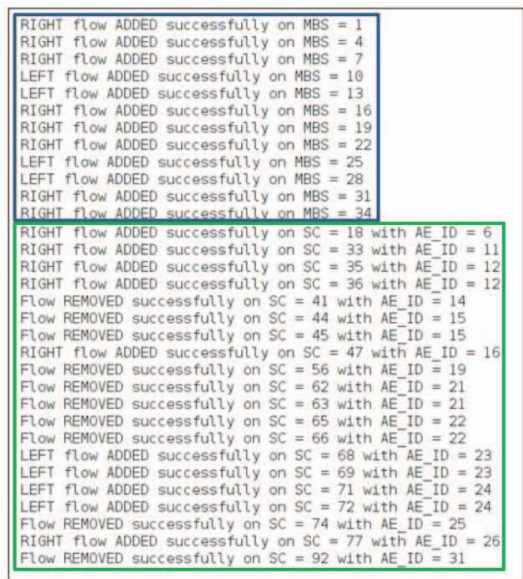

(c)

(b)

Fig. 9. Screenshots of emulation experiment for $N=2$ : (a) ODL controller adding all the nodes by reading from an XML file, (b) flow entries inside the OVSs for TPs and WSSs after sending the lambda commands from ODL, and (c) orchestrator script showing that the flows to activate/deactivate the RBSs are added/removed successfully by the ODL controller.

blue is the flow entry that is inserted in a TP when a lightpath is established and the TP works as a transmitter. This entry results in OVS adding a VLAN tag 102 to all the incoming packets at port 2 and forwarding the tagged packets to port 1 . The entry highlighted in green is the flow entry that is inserted in the WSS to switch the VLAN tagged packets. This entry results in OVS forwarding all the packets to port 5 (i.e., common port of WSS) that arrive at port 2 with a VLAN tag 102.

Figure 9(c) shows the output from the script in Java mimicking the orchestrator as it asks the ODL controller to add and remove the flows and receives a positive acknowledgment from ODL. The print statements inside the blue box depict that all the flows have been established successfully in order to activate all the MBSs in the network. These flows are added in the start of the emulation experiment and stay there throughout the lifetime of the experiment. As can be seen in the figure, each MBS and $\mathrm{SC}$ is assigned a unique ID for the sake of bookkeeping, while the AEs are assigned an ID number from 1 to 35 . This helps us to identify which RBSs are currently active in the network so that the corresponding flows can be removed when they need to be deactivated. Here, the RIGHT 
and LEFT keywords correspond to the flows that are added by traversing the metro and access rings in the clockwise and counter-clockwise directions, respectively, depending on the shorter path and availability of wavelength resources. The print statements inside the green box depict that the flows are added/removed successfully when SCs are configured during a specific hour of the experiment. For example, the SC 18 is activated by adding a RIGHT flow, while the SC 41 is deactivated by removing the flow that was active during the previous hour.

\section{B. Numerical Results}

In this subsection, we describe the simulation results (obtained using a custom MATLAB simulator) and their experimental validation (obtained using the $\mathrm{ONE}$ and the experimental setup described in Subsection VII.A).

The simulation results are obtained by running a total of 100 simulation experiments (i.e., to mimic the network operation over 100 days). We computed the average number of TPs that need to be active at the ME at each hour of the day in order to connect all the active RBSs to the BBU pool utilizing our provisioning strategy. The same experiments were also run utilizing the ONE and the experimental setup described in Subsection VII.A. However, since the experiments using the ONE are quite time consuming, we average the results over 7 experiments (i.e., to mimic the network operation over a week) instead of 100 experiments as for the simulation. Hence, there is a small difference in the simulation and emulation results, which are presented in Figs. 10 and 11. We compare the results obtained utilizing our provisioning strategy with a conventional overprovisioning approach. For the sake of simplicity, we run the simulations and emulations while only considering the upstream traffic.
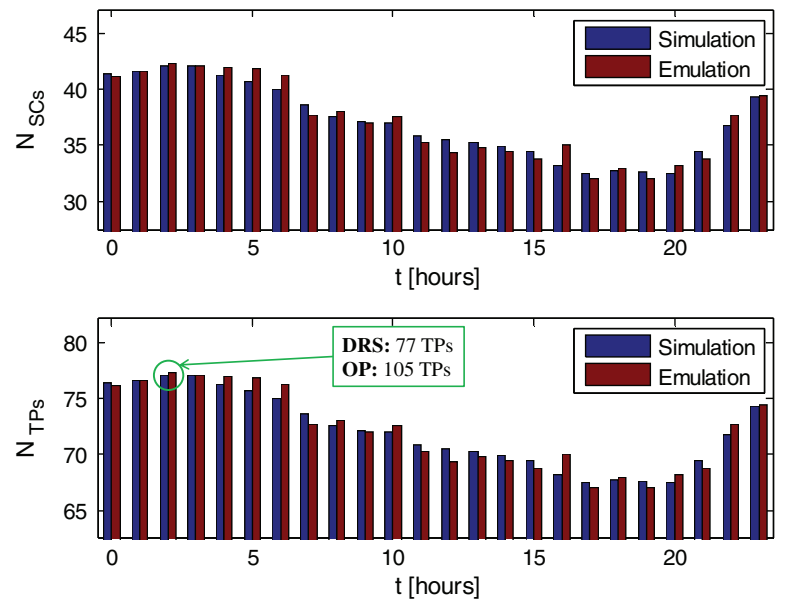

Fig. 10. Number of SCs to be activated and number of TPs in use during 24 hours of the day with $N=2 \mathrm{SCs}$ per AE by using the traffic profile in Fig. 6. DRS: dynamic resource sharing. OP: overprovisioning. $N_{\mathrm{SCs}}$ : number of active SCs. $N_{\mathrm{TPs}}$ : number of active TPs at the ME.
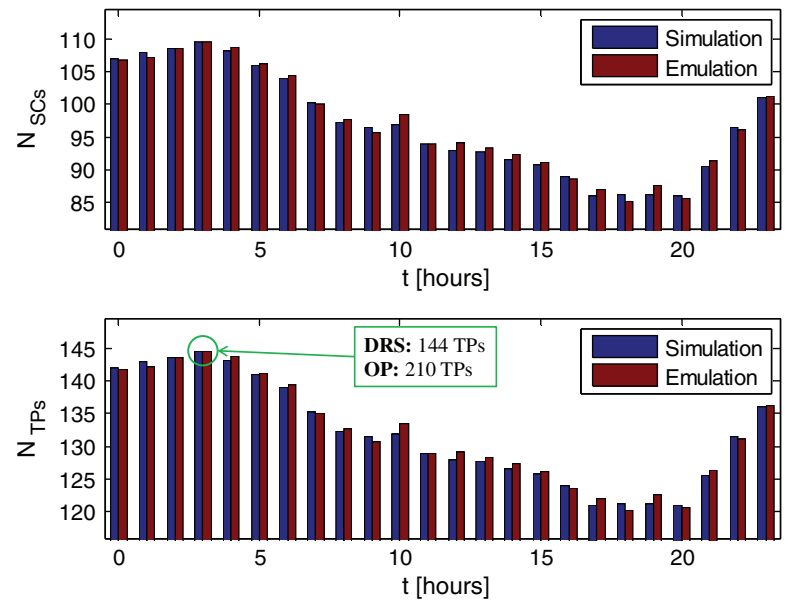

Fig. 11. Number of SCs to be activated and number of TPs in use during 24 hours of the day with $N=5$ SCs per AE by using the traffic profile in Fig. 6. DRS: dynamic resource sharing. OP: overprovisioning. $N_{\mathrm{SCs}}$ : number of active $\mathrm{SCs}$. $N_{\mathrm{TPs}}$ : number of active TPs at the ME.

The results in Figs. 10 and 11 are obtained by using the traffic profile in Fig. 6. Figure 10 shows the number of SCs $\left(N_{\mathrm{SCs}}\right)$ that need to be activated in the radio network (top) and the number of TPs $\left(N_{\text {TPS }}\right)$ that need to be active at the ME (bottom) during 24 hours in a day with $N=2 \mathrm{SCs}$ per $\mathrm{AE}$. The number of TPs includes the ones used for connecting to the SCs as well as the ones used for connecting to the MBSs. As mentioned earlier, the simulation and emulation results have a slight difference because of the difference in the number of experiments used for averaging; however, the maximum difference is limited to 1-2 TPs or SCs.

The benefits of the proposed dynamic provisioning strategy with respect to overprovisioning are quite apparent from the results in Fig. 10. In the case of overprovisioning, a total of 105 TPs (i.e., 35 for MBSs and 70 for SCs) must be deployed at the ME in order to support all the active RBSs in the network. Instead, using the proposed provisioning strategy, the number of TPs required at the ME is only 77 (i.e., the peak value at the bottom of Fig. 10). This results in a saving of $26.7 \%$ as compared to overprovisioning. Figure 11 shows similar results for $N=5 \mathrm{SCs}$ per AE. In this case, the access ring traffic profiles are scaled to provide a maximum capacity of $1.75 \mathrm{Gbps} / \mathrm{km}^{2}$. Here, the number of TPs that needs to be deployed at the ME using our dynamic provisioning strategy is 144 (i.e., the peak value in the bottom of Fig. 11) as compared to 210 (i.e., 35 for MBSs and 175 for SCs) required using overprovisioning. This results in a saving of $31.4 \%$ in terms of number of TPs.

Figure 12 presents a set of results obtained with the traffic profiles shown in Fig. 7. In this case, we present only simulation results because emulation experiments require a long runtime and lead to very similar trends (as can be observed in Figs. 10 and 11). Figure 12 (top) shows the number of TPs that need to be active at different times of the day at the ME with $N=2 \mathrm{SCs}$ per AE. Using the proposed provisioning strategy, the number of TPs that 

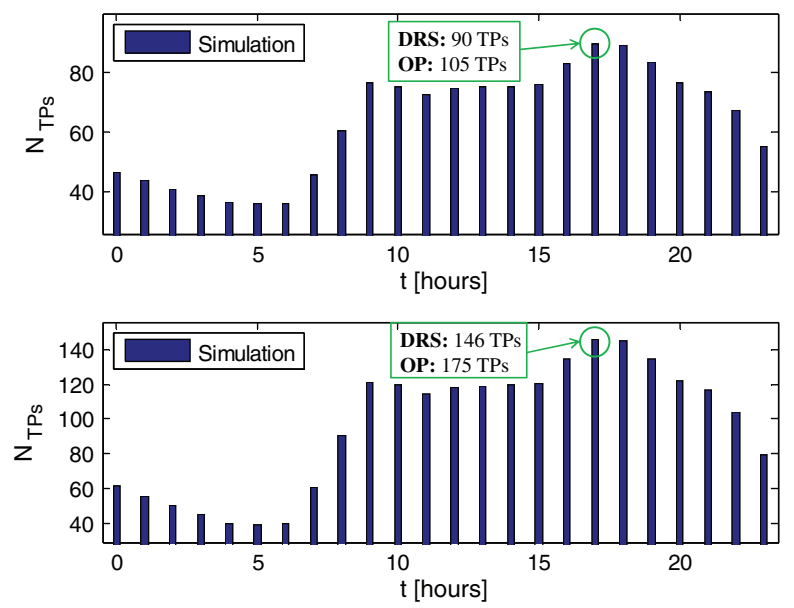

Fig. 12. Number of TPs in use during 24 hours of the day with $N=2 \mathrm{SCs}$ per AE (top) and $N=4 \mathrm{SCs}$ per AE (bottom) by using the traffic profiles in Fig. 7. DRS: dynamic resource sharing. OP: overprovisioning. $N_{\text {TPs }}$ : number of active TPs at the ME.

need to be deployed at the ME is 90 (i.e., peak value in Fig. 12, top) as compared to 105 TPs required for the case of overprovisioning, i.e., a savings of $14.3 \%$. Figure 12 (bottom) shows similar results obtained with $N=4$ SCs per $\mathrm{AE}$. In this case, the traffic profiles are scaled to provide a maximum capacity of $1.5 \mathrm{Gbps} / \mathrm{km}^{2}$. The saving in the number of TPs for this case is $16.6 \%$ (i.e., 146 TPs for dynamic resource sharing as compared to 175 TPs for overprovisioning).

It can be observed that the savings achieved using the traffic profiles in Fig. 7 (see Fig. 12) are lower with respect to the savings obtained using the traffic profile in Fig. 6 (see Figs. 10 and 11). This is mainly because the three daily traffic profiles for the different geo-types in Fig. 7 present high traffic requirements during the evening time (e.g., from 16:00 to 19:00). As a consequence, the number of TPs that need to be activated during this time of the day is high, i.e., close to the maximum needed in the case of overprovisioning. On the other hand, by using the traffic profile in Fig. 6 with a shift of three hours among the access rings, the daily traffic requirements are more randomized. As a consequence, there is no time of day in which a very large number of TPs needs to be active, and the achievable savings with respect to overprovisioning are higher.

In the following, we compare the total cost of the transport infrastructure obtained by using our proposed provisioning strategy and the overprovisioning approach. Due to space limitation, the cost analysis is presented here only for the case with the traffic profile in Fig. 6 . We perform the comparison as a function of the number of SCs per $\mathrm{AE}(N)$.

Using the dynamic provisioning strategy, we observe that when increasing $N$ beyond 5, we might not be able to always connect all the active SCs to the BBU pool. This is due to the fact that during peak traffic situations, our strategy might not be able to find enough wavelength resources in the transport network to connect all the active SCs. Consequently, the activation of some SCs might not be possible, leading to some of the activation requests being blocked. If the network operator wants to deploy more than 5 SCs per AE without any blocking (i.e., with blocking probability $P_{\text {block }}=0$ ), the only option is to add another fiber in the transport network. This in turn requires adding another layer of WSSs to the ones already deployed, driving up the total cost of the transport infrastructure.

For the case of overprovisioning, it is found that there is a need for adding a new layer of WSSs in the infrastructure for $N=5$. Since in this case, 1 wavelength is reserved for MBS and 5 wavelengths are reserved for SCs, so a total of 210 lightpaths is required to activate all the RBSs in 35 AEs. Since each fiber is considered to support 96 wavelengths (which can be used in both directions, i.e., clockwise and counter-clockwise), we can have up to 192 lightpaths established in the network, which is not sufficient for $N=5$. Hence, by using the overprovisioning strategy, a new layer of WSSs needs to be added for the value of $N$ beyond 4. Moreover, if $N$ is increased further, the number of wavelengths may no longer be sufficient at a certain value of $N$, and there will be a need for adding again a new layer of WSSs.

We increase the value of $N$ from 2 to $20 \mathrm{SCs}$ per AE, and for each value of $N$, we compute the total cost of the transport network by counting the total number of TPs and WSS ports required in the network. The results are shown in Fig. 13 (blue and green curves). For each value of $N$, the access ring traffic profiles are scaled accordingly. We assume that the price of a $10 \mathrm{Gbps}$ TP is 1 cost unit (CU), and the price per port of a WSS is 0.78 CUs. This assumption is based on the cost values for optical equipment for the year 2018 reported in Ref. [26]. Note that for simplicity, we assume that the cost of a WSS depends linearly on the number of ports [26]. The points in which a new layer of WSSs needs to be added to the transport network can be observed by sudden jumps of the total cost in Fig. 13.

It can be observed by comparing the green and blue curves in Fig. 13 that a significant amount of cost savings can be achieved by using our strategy based on dynamic resource sharing as compared to overprovisioning. The

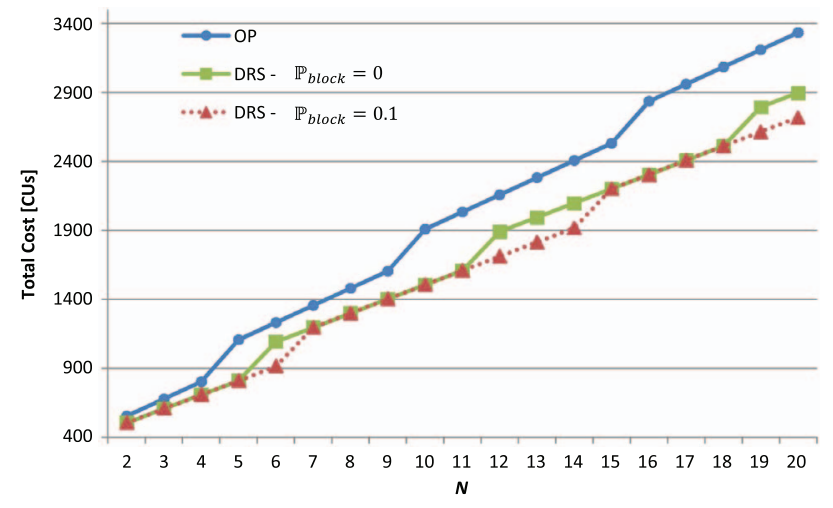

Fig. 13. Total cost of the network versus number of SCs per AE $(N)$. The cost is compared for the cases of overprovisioning (OP) and dynamic resource sharing (DRS) strategies. $\mathbb{P}_{\text {block }}$ : blocking probability. 
maximum amount of cost savings is $18.6 \%$, which can be observed at $N=18$. This comes mainly from savings in the number of TPs connected to the ME, which also results in a reduction in the number of ports of WSSs in the ME. Second, the proposed dynamic approach can help to add a few more SCs without the need for an extra layer of WSSs. Hence, the need for increasing the amount of WSSs appears at a higher value of $N$ as compared to overprovisioning, which also leads to a cost savings at the corresponding values of $N$, as shown by the green curve in Fig. 13 .

In the analysis done so far, we assumed that the network operator does not accept that the activation of an SC can be blocked during peak traffic situations because of the lack of wavelength resources (i.e., we assumed that the operator allows only blocking probability $P_{\text {block }}=0$ ). However, in some scenarios, e.g., with very dense SC networks, it might be acceptable to tolerate some blocking probability during peak traffic situations (note that we assume that only SCs can be blocked, while MBSs are always active to guarantee wireless coverage). In order to evaluate the performance for such a scenario, we define the blocking probability as the ratio of number of the SCs that cannot be activated (i.e., due to lack of wavelengths) to the total number of SCs that need to be activated to satisfy the current wireless traffic demand. Assuming that the maximum acceptable value of blocking probability for the network operator is $P_{\text {block }}=0.1$, we repeat the cost calculations for the case of provisioning with dynamic resource sharing. The results are shown in Fig. 13 (red curve). It can be seen that the red and green curves are quite close, with the only difference coming from the value of $N$ where there is a need for duplication of the transport infrastructure. Under such a scenario with an acceptable value of blocking probability, the network operator can add a few more SCs without the need for adding a new layer of WSSs and hence gets an opportunity for some cost savings. For example, the case without blocking probability (green curve) requires adding a new layer of WSSs at $N=12$, while the case with blocking probability of 0.1 (red curve) requires a new layer of WSSs only for $N=15$. Hence, the network operator can add three more SCs without any significant difference in the total cost of the network.

\section{CONCLUSION}

This paper proposes a provisioning strategy based on dynamic resource sharing for a C-RAN with optical transport. It relies on the use of a hierarchical SDN control plane where a global orchestrator harmonizes the use of the transport resources according to the spatial and temporal variations of the wireless traffic. Moreover, an optical network emulator has been developed and used to prove the benefits of the proposed provisioning strategy as well as for testing the scalability of the hierarchal SDN control plane framework proposed in Ref. [7] in a realistic network scenario.

The simulation and emulation results presented in the paper confirm the initial intuition that the joint orchestration of radio and transport resources may lead to a more efficient use of the transport resources. In fact, with the proposed provisioning strategy, it is possible to achieve up to $31.4 \%$ of savings in the number of optical transponders when compared to a conventional overprovisioning strategy. This translates in significant cost savings for network operators. Much higher cost savings can be expected at the moment when network operators plan to deploy even more small cells in the network, as dictated by most of the envisioned 5G traffic scenarios. As a future study, it would be interesting to investigate a data plane architectural solution able to provide sufficient flexibility to allow the use of dynamic resource sharing while at the same time reducing the cost by maximizing the use of passive equipment.

\section{ACKNOWLEDGMENT}

The authors would like to acknowledge Joakim Lindberg for his contribution in the development of the optical network emulator. The work described in this paper was carried out with the support of the Kista 5G Transport Lab (K5) project funded by VINNOVA and Ericsson.

\section{REFERENCES}

[1] P. Ohlen, B. Skubic, A. Rostami, M. Fiorani, P. Monti, Z. Ghebretensae, J. Mårtensson, K. Wang, and L. Wosinska, "Data plane and control architectures for 5G transport networks," IEEE J. Lightwave Technol., vol. 34, no. 6, pp. 1501-1508, Mar. 2016.

[2] J. Andrews, S. Buzzi, W. Choi, S. V. Hanly, A. Lozano, A. C. K. Soong, and J. C. Zhang, "What will 5G be?" IEEE J. Sel. Areas Commun., vol. 32, no. 6, pp. 1065-1082, June 2014.

[3] "5G radio access," Ericsson White Paper, Apr. 2016 [Online]. Available: https://www.ericsson.com/res/docs/whitepapers/wp5g.pdf.

[4] P. Rost, C. J. Bernardos, A. D. Domenico, M. D. Girolamo, M. Lalam, A. Maeder, D. Sabella, and D. Wuebben, "Cloud technologies for flexible $5 \mathrm{G}$ radio access networks," IEEE Commun. Mag., vol. 52, no. 5, pp. 68-76, May 2014.

[5] M. Fiorani, P. Monti, B. Skubic, J. Mårtensson, L. Valcarenghi, P. Castoldi, and L. Wosinska, "Challenges for 5G transport networks," in IEEE Conf. on Advanced Networks and Telecommunication Systems (ANTS), Dec. 2014.

[6] H. Wang, F. Xu, Y. Li, P. Zhang, and D. Jin, "Understanding mobile traffic patterns of large scale cellular towers in urban environment," in ACM Internet Measurement Conf. (IMC), 2015.

[7] A. Rostami, K. Wang, Z. Ghebretensae, P. Ohlen, and B. Skubic, "First experimental demonstration of orchestration of optical transport, RAN and cloud based on SDN," in Optical Fiber Communications Conf. and Exhibition (OFC), Mar. 2015.

[8] A. Mayoral, R. Vilalta, R. Munoz, R. Casellas, R. Martinez, and J. Vilchez, "Integrated IT and network orchestration using OpenStack, OpenDaylight and active stateful PCE for intra and inter data center connectivity," in European Conf. on Optical Communication (ECOC), Sept. 2014.

[9] A. Mayoral, R. Vilalta, R. Munoz, R. Casellas, and R. Martinez, "Experimental seamless virtual machine migration using an integrated SDN IT and network orchestrator," in Optical Fiber Communications Conf. and Exhibition (OFC), Mar. 2015.

[10] R. Muñoz, R. Vilalta, R. Casellas, R. Martinez, T. Szyrkowiec, A. Autenrieth, V. López, and D. López, "Integrated SDN/NFV 
management and orchestration architecture for dynamic deployment of virtual SDN control instances for virtual tenant networks [Invited]," J. Opt. Commun. Netw., vol. 7, pp. B62-B70, 2015.

[11] B. Sonkoly, J. Czentye, R. Szabo, D. Jocha, J. Elek, S. Sahhaf, W. Tavernier, and F. Risso, "Multi-domain service orchestration over networks and clouds: A unified approach," in Proc. ACM SIGCOMM, Aug. 2015.

[12] "EU project UNIFY, Unifying cloud and carrier networks" [Online]. Available: http://www.fp7-unify.eu/.

[13] J. Costa-Requena, J. L. Santos, and V. F. Guasch, "Mobile backhaul transport streamlined through SDN," in Int. Conf. on Transparent Optical Networks (ICTON), July 2015.

[14] J. Costa-Requena, V. Ferrer Guasch, and J. Llorente Santos, "Software defined networks based 5G backhaul architecture," in ACM Proc. Int. Conf. on Ubiquitous Information Management and Communication (IMCOM), Jan. 2015.

[15] M. Fiorani, A. Rostami, L. Wosinska, and P. Monti, "Transport abstraction models for an SDN-controlled centralized RAN," IEEE Commun. Lett., vol. 19, no. 8, pp. 1406-1409, Aug. 2015.

[16] A. Rostami, P. Öhlén, K. Wang, Z. Ghebretensaé, and B. Skubic, "Orchestration of RAN and transport networks resources for 5G: An SDN approach," IEEE Commun. Mag., to be published.

[17] "C-RAN-The road towards green RAN," China Mobile White Paper, Version 3.0, Dec. 2013 [Online]. Available: http://labs.chinamobile.com/cran/wp-content/uploads/2014/ 06/20140613-C-RAN-WP-3.0.pdf.
[18] "OpenDaylight, A Linux foundation collaborative project" [Online]. Available: http://www.opendaylight.org/.

[19] "Open vSwitch, An open source multi-layer virtual switch" [Online]. Available: http://www.openvswitch.org/

[20] "Mininet, A network emulation software" [Online]. Available: http://www.mininet.org/

[21] N. McKeown, T. Anderson, H. Balakrishnan, G. Parulkar, L. Peterson, J. Rexford, S. Shenker, and J. Turner, "OpenFlow: Enabling innovation in campus networks," Comput. Commun. Rev., vol. 38, no. 2, pp. 69-74, Apr. 2008.

[22] "POX, an open source OpenFlow controller" [Online]. Available: http://www.noxrepo.org

[23] S. Tombaz, P. Frenger, F. Athley, E. Semaan, C. Tidestavand, and A. Furuskär, "Energy performance of 5G-NX wireless access utilizing massive beamforming and an ultra-lean system design," in IEEE Global Communications Conf. (GLOBECOM), 2015.

[24] M. Fiorani, S. Tombaz, J. Mårtensson, B. Skubic, L. Wosinska, and P. Monti, "Energy performance of C-RAN with 5G-NX radio networks and optical transport," in IEEE Int. Conf. on Communications (ICC), May 2016.

[25] "Energy efficiency analysis of the reference systems, areas of improvements and target breakdown," EARTH Deliverable D2.3, 2012.

[26] M. R. Raza, M. Fiorani, P. Monti, B. Skubic, J. Mårtensson, and L. Wosinska, "Power and cost modeling for 5G transport networks," in IEEE Int. Conf. on Transparent Optical Networks (ICTON), July 2015. 